\title{
Whole exome sequencing of microdissected splenic marginal zone lymphoma: a study to discover novel tumor-specific mutations
}

\author{
Jan Peveling-Oberhag ${ }^{1 *}$, Franziska Wolters ${ }^{1}$, Claudia Döring $^{2}$, Dirk Walter ${ }^{1}$, Ludger Sellmann ${ }^{3}$, René Scholtysik ${ }^{3}$, \\ Marco Lucioni ${ }^{4}$, Max Schubach ${ }^{5}$, Marco Paulli ${ }^{4}$, Saskia Biskup ${ }^{6}$, Stefan Zeuzem ${ }^{1}$, Ralf Küppers ${ }^{3,7}$ and \\ Martin-Leo Hansmann ${ }^{2,7}$
}

\begin{abstract}
Background: Splenic marginal zone lymphoma (SMZL) is an indolent B-cell non-Hodgkin lymphoma and represents the most common primary malignancy of the spleen. Its precise molecular pathogenesis is still unknown and specific molecular markers for diagnosis or possible targets for causal therapies are lacking.

Methods: We performed whole exome sequencing (WES) and copy number analysis from laser-microdissected tumor cells of two primary SMZL discovery cases. Selected somatic single nucleotide variants (SNVs) were analyzed using pyrosequencing and Sanger sequencing in an independent validation cohort.

Results: Overall, 25 nonsynonymous somatic SNVs were identified, including known mutations in the NOTCH2 and MYD88 genes. Twenty-three of the mutations have not been associated with SMZL before. Many of these seem to be subclonal. Screening of 24 additional SMZL for mutations at the same positions found mutated in the WES approach revealed no recurrence of mutations for ZNF608 and PDE10A, whereas the MYD88 L265P missense mutation was identified in $15 \%$ of cases. An analysis of the NOTCH2 PEST domain and the whole coding region of the transcription factor SMYD1 in eight cases identified no additional case with a NOTCH2 mutation, but two additional cases with SMYD1 alterations.

Conclusions: In this first WES approach from microdissected SMZL tissue we confirmed known mutations and discovered new somatic variants. Recurrence of MYD88 mutations in SMZL was validated, but NOTCH2 PEST domain mutations were relatively rare (10\% of cases). Recurrent mutations in the transcription factor SMYD1 have not been described in SMZL before and warrant further investigation.
\end{abstract}

Keywords: Splenic marginal zone lymphoma, Lymphoma, Next generation sequencing, SMYD1, NOTCH2

\section{Background}

Splenic marginal zone lymphoma (SMZL) is a rare lowgrade B-cell lymphoma listed as distinct entity in the World Health Organization (WHO) classification of lymphoid neoplasm, accounting for less than $2 \%$ of non-Hodgkin lymphomas $[1,2]$. It commonly follows an indolent course exceeding a median 10-year survival. However, in a minority of cases it can pursue a more

\footnotetext{
* Correspondence: jan.peveling-oberhag@kgu.de

${ }^{1}$ Medizinische Klinik 1, Klinikum der Johann Wolfgang Goethe-Universität,

Theodor-Stern-Kai 7, Frankfurt am Main, Germany

Full list of author information is available at the end of the article
}

aggressive course with the possibility of transformation into a diffuse large B-cell lymphoma [3].

The molecular pathogenesis of SMZL is still not entirely clear. There are no signature genetic mutations which allow a reliable clinical diagnosis. Cytogenetic studies in the past identified recurrent deletions of $7 q 22-q 36$ and $3 q$ gains in approx. $45-50 \%$ and $20-30 \%$ of cases, respectively [4]. However, within these regions, molecular studies did not identify single genes with an essential role in SMZL pathogenesis $[5,6]$. Candidate gene studies, gene expression profiling or miRNA expression studies revealed recurring molecular signatures such as the NF- $\mathrm{kB}$ pathway or increased B-cell receptor signaling [7-10]. Several

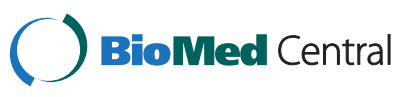

(c) 2015 Peveling-Oberhag et al. Open Access This article is distributed under the terms of the Creative Commons Attribution 4.0 International License (http://creativecommons.org/licenses/by/4.0/), which permits unrestricted use, distribution, and reproduction in any medium, provided you give appropriate credit to the original author(s) and the source, provide a link to the Creative Commons license, and indicate if changes were made. The Creative Commons Public Domain Dedication waiver (http://creativecommons.org/publicdomain/zero/1.0/) applies to the data made available in this article, unless otherwise stated. 
whole-genome sequencing- or whole-exome sequencing (WES) studies identified recurrent somatic mutations involved in the NF- $\mathrm{kB}$ pathway [11-14]. Mutations in NOTCH2, which eliminate the C-terminal PEST domain and result in compromised protein degradation, were identified in $20-25 \%$ of cases in two studies [11, 14], but such high frequencies were not confirmed in other investigations [12, 13]. Recently, mutations in KLF2, a member of the Krüppel-like family of transcription factors with roles in cell differentiation, proliferation, activation and trafficking were found recurrently mutated in SMZL. First studies showed high frequency of these mutations in up to $40 \%$ of SMZL cases $[15,16]$, but a consecutive study found lower SNV-frequencies (12\% of cases) [17]. The tumor DNA investigated in the previous studies was retrieved either from whole frozen tissue or paraffine embedded sections or $\mathrm{CD} 19^{+}$cells from the peripheral blood or tumor tissue.

In our current study we performed WES from lasermicrodissected tumor cells deriving from two cases of SMZL. We confirm several somatic mutations from previous studies and expand the known genetic signature of SMZL by several newly discovered mutations.

\section{Methods}

\section{Patient selection}

Splenic tissue from 26 patients with SMZL was selected for this study from the Department of Pathology, Pavia, Italy, the Department of Pathology, Frankfurt, Germany, and the University of Duisburg-Essen, Medical School, Essen, Germany. Two splenic tissue samples were used to perform next generation sequencing and were selected for their classical tumor morphology, high tumor cell content and availability of fresh tissue (Table 1). For validation experiments, samples of 24 patients were selected who underwent splenectomy for SMZL and featured a tumor cell content of greater than $60 \%$ (estimated by morphology and immunohistochemistry; summary of clinical data presented in Additional file 1: Table S1). Within the validation group, fresh frozen tissue as well as formalinfixed, paraffin-embedded (FFPE) tissue was available in 8 patients. In the remaining 16 patients, only FFPE tissue was available. The diagnosis of SMZL was established by standard morphological, cytochemical and immunophenotypic methods according to the 2008 WHO lymphoma classification and its diagnostic criteria [2, 18]. All cases included in the study were classical SMZL, with a typical $\mathrm{CD} 5^{-}, \mathrm{CD} 10^{-}, \mathrm{Bcl}-6^{-}, \mathrm{CD} 23^{-}$phenotype and with a typical pattern of white pulp involvement. Patient samples that showed MYD88 mutations in the current sequencing analysis were re-evaluated to confirm SMZL diagnosis. Informed patient consent was obtained according to the declaration of Helsinki, and the study was ethically approved by the ethics committee of the Medical faculty of the Goethe-University of Frankfurt (Vote \#4/09, 2013).

\section{DNA isolation from tumor cells and non-tumorous controls}

For WES, tumor cells were specifically laser-microdissected from fresh frozen tissue sections as described elsewhere [9]. After hemalaun and eosin staining, areas of tumor cells within the splenic marginal zone were selectively microdissected and the tissue was directly transferred into DNA lysis buffer. Representative counting of neoplastic cells within microdissected tissue showed mean tumor cell proportions between 87 and $89 \%$. The co-analyzed non-tumorous controls were purified from fresh splenic tissue of the corresponding patient using MACS-sorting for $\mathrm{CD}^{+}$cells. For validation, DNA was isolated from whole tissue slides. DNA purification was performed using the QIAamp DNA extraction kit (QIAGEN, Hilden, Germany).

\section{Whole exome enrichment and sequencing}

Purified tumor and germline genomic DNA $(3 \mu \mathrm{g})$ from the two discovery SMZL cases was enriched in protein coding sequences using the in-solution exome capture SureSelect Human All Exon $50 \mathrm{Mb}$ kit (Agilent Technologies, Böblingen, Germany), according to the manufacturer's protocol. WES was performed using the SOLiD4 Platform (Life Technologies, Darmstadt, Germany). Each sample was sequenced on a single quad of a SOLiD sequencing slide (Life Technologies).

\section{Single nucleotide polymorphism (SNP)-array analysis}

Tumor and germ-line DNA was purified, amplified, labelled and hybridized to the Affymetrix SNP5.0 platform (Affymetrix, Santa Clara, CA) according to the manufacturer's protocol. Copy number profiles (aligned to hg19/ GRCh37) from tumor and germ-line samples were compared using the Genotyping Console software (Affymetrix). The minimum number of SNP-markers per segment were

Table 1 Clinical characteristics of discovery case 1 and 2

\begin{tabular}{|c|c|c|c|c|c|c|c|}
\hline $\begin{array}{l}\text { Discovery } \\
\text { case }\end{array}$ & Sex & $\begin{array}{l}\text { Age at } \\
\text { splenectomy }\end{array}$ & B-Symptoms & $\begin{array}{l}\text { Leukemic disease } \\
\text { (villous Lymphozytes) }\end{array}$ & Immunophenotype & $\begin{array}{l}\text { Clinical staging } \\
\text { at splenectomy }\end{array}$ & $\begin{array}{l}\text { Oncological } \\
\text { response }^{a}\end{array}$ \\
\hline 1 & $f$ & 59 & yes & no & $\begin{array}{l}\text { CD20+++, IgM-, Kappa-, Lambda-, Ki-67 } 5 \text { \%, } \\
\text { CD5-, CD3-, Cyclin-D1-, bcl-2++ }\end{array}$ & IV & $C R$ \\
\hline 2 & $f$ & 59 & yes & no & $\begin{array}{l}\text { CD20+++, IgM+, Kappa-, Lambda-, Ki-67 } \\
15 \% \text {, CD5-, CD3-, Cyclin-D1-, bcl-2++ }\end{array}$ & IV & $C R$ \\
\hline
\end{tabular}

after splenectomy 
set to 5 with a minimum genomic size of a segment of $100 \mathrm{~kb}$. Allele ratio and copy number neutral loss of heterozygosity were calculated for each sample using the HapMap Allele Reference baseline (Affymetrix).

\section{Validation with pyrosequencing and Sanger sequencing}

The pyrosequencing technique was used for validation of specific discovered single base substitution-type mutations (NOTCH2 C7310T, SMYD1 G839T, MYD88 T794C, ZNF608 A3659G, PDE10A G1072A) in a validation cohort of 24 microdissected SMZL samples. An internal fragment of each gene was amplified by polymerase chain reaction (PCR) using primers specific for each gene and a PyroMark PCR kit (QIAGEN). The resulting PCR products were sequenced with the PyroMark Q24 (QIAGEN) pyrosequencer using PyroMark Gold Q96 reagents (QIAGEN) and sequencing primers specific for each gene.

Conventional Sanger sequencing was used to screen for mutations in two selected genes within the validation cohort (NOTCH2 and SMYD1). The PEST domain of $\mathrm{NOTCH} 2$ within exon 34 and all 10 exons of SMYD were sequenced in 8 validation and 2 WES cases. Primer sequences are given in Additional file 2: Table S2; Additional file 3: Table S3; Additional file 4: Table S4.

\section{Bioinformatics and statistical analyses}

Next generation sequencing reads were mapped with the Blast-like mapping algorithm from Bioscope v1.2 (Life Technologies) against the human reference genome (hg19 from UCSC) using color codes. High sensitive variant calling-including small insertions and deletions as well as single nucleotide variants (SNVs)-was performed by the DiBayes algorithm from Bioscope. Transcript and protein alterations were annotated with NGS-SNP [19] using the ENSEMBL v61 database [20, 21]. Only variants potentially changing the protein sequence were used for further analysis; intronic, UTR and synonymous mutations were removed. In addition, low quality SNVs were filtered out using the novel allele mean quality given by Bioscope. All SNVs that were below the mean minus two times the standard deviation from a calling were discarded.

To find somatic variants, only SNVs with a minimum coverage of 20 and a minimum novel allele frequency of 0.1 were intersected with the variants from the normal tissue. Somatic variants were called if the allele frequency of the normal tissue was smaller 0.2 and the delta between tumor and normal frequency was at least 0.1. All SNVs identified by this algorithm underwent manual review by two independent observers using the integrative genomics viewer (Broad Institute, v1.5) to reduce the rate of false positives [22]. Protein illustration was performed using ballView software (http:// www.ballview.org/).

\section{Results}

Two well-matched SMZL patients (both female, same age at splenectomy, similar morphology and immunophenotype of SMZL; Table 1) were chosen for NGS analysis. Exome-capture and high-throughput sequencing of microdissected lymphoma cells from two SMZL allowed us to align approx. 62.1 million reads per sample with an off-target read number of 3.4 million $(5.5 \%)$ at a mean depth of 47-fold (range 42-49). In total, an average of 77, 66 and $54 \%$ of target sequences were captured at a minimum coverage of 10,20 and 30 , respectively. Our analytical algorithm identified 216 non-synonymous variants (Additional file 5: Table S5). Of these, 191 were filtered out, as they were either identified as previously described polymorphisms or showed high incidence in the non-tumorous matched controls, leaving 25 probable somatic mutations, 12 in case 1 and 13 in case 2 (Table 2). Of the 25 base substitutions, 23 were missense mutations, one was a splice site, and one was a nonsense mutation (Fig. 1a). In addition 64 and $36 \%$ of these variants were transitions and transversions, respectively (Fig. 1b).

SNP-array analysis of the two SMZL identified 3 somatically-acquired copy number aberrations. Case 2 features a duplication of $3 \mathrm{q}$ and both cases harbour a deletion in 7q (Fig. 2), which in both cases covers the $7 \mathrm{q} 32$ region previously described in SMZL $[4,6]$. Only one of the mutations identified (MYD88 L265P) falls within a copy number aberration, namely the 3q gain. The MYD88 L265P mutation in the discovery case with the 3q duplication was called in only one third of the reads (14 of 50) by the WES analysis, so likely the mutation is located on the non-duplicated region of chromosome 3.

Somatic mutations identified in the discovery cases are shown in Table 2. Only two of these (NOTCH2 and MYD88) have been described in SMZL previously [11-14, 23-25]. Twenty-three variants discovered in the current investigation have, to our knowledge, not been described in SMZL before. None of these mutations was found in both cases. Furthermore, none of the somatic mutations showed features of homozygosity (70-100\% frequency of the mutated variant) and only 9 mutations (36\%) showed a minor allele call of $>35 \%$. SIFT algorithm predicted about half of the mutations to be damaging (10/25 variants damaging, $1 / 25$ stop gained, Table 2) [26]. Similar predictions were made by the Polyphen-2 algorithm [27], which classified $13 / 25$ variants to be possibly or probably damaging (Table 2). As mutations in KLF2 have been found with high frequencies in SMZL in other studies, this region was specifically reviewed within the current WES data. The bioinformatic SNP-detection algorithm did not detect any mutations in this area. Also manual review of the region 
Table 2 Somatic mutations in the two SMZL analyzed by WES

\begin{tabular}{|c|c|c|c|c|c|c|c|c|c|c|c|}
\hline Gene & Case & Chr & Position & Exon & $\begin{array}{l}\text { Coverage } \\
\text { tumor }\end{array}$ & $\begin{array}{l}\text { SNV } \\
\text { frequency }\end{array}$ & $\begin{array}{l}\text { Quality } \\
\text { score }^{\mathrm{a}}\end{array}$ & $\begin{array}{l}\text { Nucleotide } \\
\text { change }\end{array}$ & $\begin{array}{l}\text { AA } \\
\text { change }\end{array}$ & $\begin{array}{l}\text { SIFT } \\
\text { prediction }\end{array}$ & $\begin{array}{l}\text { PolyPhen-2 } \\
\text { prediction }\end{array}$ \\
\hline CEBPZ & 1 & 2 & 37.456 .150 & 2 & 21 & 0.38 & 27.0 & c.186 T>A & p.D62E & Tolerated & Benign \\
\hline MYD88 & 1 & 3 & 38.182 .641 & 5 & 49 & 0.49 & 30.0 & C.794 T>C & p.L265P & Damaging & Probably damaging \\
\hline TTC14 & 1 & 3 & 180.320 .802 & 2 & 21 & 0.24 & 32.0 & c. $285 A>G$ & p.E95E ${ }^{b}$ & Tolerated & Benign \\
\hline SLC6A7 & 1 & 5 & 149.574 .417 & 2 & 33 & 0.27 & 23.0 & C.160 T > C & p.C54R & Damaging & Probably damaging \\
\hline BTN2A2 & 1 & 6 & 26.384 .057 & 2 & 29 & 0.24 & 32.0 & $\mathrm{C} .8 \mathrm{C}>\mathrm{T}$ & p.P3L & Damaging & Possibly damaging \\
\hline POM121 & 1 & 7 & 72.396 .892 & 5 & 41 & 0.29 & 20.0 & c. $737 \mathrm{C}>\mathrm{T}$ & p.P246L & Damaging & Probably damaging \\
\hline MUC12 & 1 & 7 & 100.645 .822 & 2 & 32 & 0.25 & 31.0 & c. 12407 C > A & p.P4136H & Tolerated & Probably damaging \\
\hline STMN4 & 1 & 8 & 27.098.767 & 5 & 40 & 0.25 & 30.0 & c. $203 A>C$ & p.D68A & Damaging & Benign \\
\hline KRTAP5-2 & 1 & 11 & 1.619 .441 & 1 & 20 & 0.35 & 27.0 & C.40 T >C & p.C14R & N/A & Possibly damaging \\
\hline CACNA1C & 1 & 12 & 2.794 .937 & 47 & 63 & 0.25 & 20.0 & c. $5823 C>T$ & p.T1941M & Tolerated & N/A \\
\hline LOC728888 & 1 & 16 & 29.395.118 & & 61 & 0.15 & 18.0 & c. $1135 G>T$ & p.G379W & Damaging & Probably damaging \\
\hline CDC27 & 1 & 17 & 45.247 .333 & 4 & 42 & 0.26 & 28.0 & c. $327 \mathrm{G}>\mathrm{T}$ & p.E109D & Tolerated & Benign \\
\hline FBXO44 & 2 & 1 & 11.718 .850 & 4 & 21 & 0.19 & 20.28 & $c .421 \mathrm{G}>\mathrm{A}$ & p.V1411 & Tolerated & Benign \\
\hline $\mathrm{NOTCH} 2$ & 2 & 1 & 120.458 .255 & 34 & 65 & 0.48 & 22.0 & c.7090C > T & p.Q2364 & Stop gained & N/A \\
\hline SMYD1 & 2 & 2 & 88.396 .251 & 6 & 26 & 0.50 & 26.0 & $c .836 \mathrm{G}>\mathrm{T}$ & p.C279F & Damaging & Probably damaging \\
\hline ZNF608 & 2 & 5 & 123.982 .418 & 4 & 73 & 0.33 & 26.0 & c. $3659 A>G$ & p.D1220G & Damaging & Probably damaging \\
\hline ZNF451 & 2 & 6 & 57.013 .380 & 10 & 30 & 0.20 & 24.93 & c. $2497 \mathrm{~T}>\mathrm{C}$ & p.F833L & Tolerated & Benign \\
\hline PDE10A & 2 & 6 & 165.829 .696 & 13 & 116 & 0.37 & 22.0 & c. $1072 \mathrm{G}>\mathrm{A}$ & p.A358T & Tolerated & Probably damaging \\
\hline PCLO & 2 & 7 & 82.784 .519 & 2 & 57 & 0.18 & 17.0 & c. $1438 C>T$ & p.P480S & Tolerated & Benign \\
\hline PRSS1 & 2 & 7 & 142.460 .369 & 4 & 26 & 0.23 & 19.0 & $c .542 \mathrm{G}>\mathrm{A}$ & p.S181N & Tolerated & Benign \\
\hline CSMD1 & 2 & 8 & 2.800 .055 & 69 & 74 & 0.36 & 24.0 & $c .8683 \mathrm{~A}>\mathrm{T}$ & p.12895F & Damaging & N/A \\
\hline APOA4 & 2 & 11 & 116.692 .500 & 3 & 20 & 0.35 & 31.0 & c. $274 G>A$ & p.A92T & Tolerated & Benign \\
\hline HERC2 & 2 & 15 & 28.458 .971 & 42 & 23 & 0.22 & 25.97 & c. $6703 \mathrm{C}>\mathrm{A}$ & p.R2235s & Tolerated & Possibly damaging \\
\hline CDC27 & 2 & 17 & 45.229 .185 & 9 & 90 & 0.23 & 29.0 & c. $1075 G>C$ & p.G359R & Tolerated & Possibly damaging \\
\hline GRIN2C & 2 & 17 & 72.842 .984 & 10 & 39 & 0.44 & 23.0 & c. $2077 C>T$ & p.R693C & Damaging & Probably damaging \\
\hline
\end{tabular}

AA amino acid, Chr chromosome, N/A not applicable, SIFT Sorting Tolerant from Intolerant Algorithm, SNV[space]single nucleotide variant

${ }^{a}$ calculated by Bioscope algorithm

${ }^{b}$ splice site

'Stop gained

of interest did not detect KLF2 mutations. However, large areas of this gene showed poor coverage (mean coverage, range: $12.6,1-44$ ), although the enrichment design included specific baits for this region. The coverage statistics of other selected genes of interest mentioned in this study are shown in Additional file 6: Table S6.

Five of the 25 genes were chosen for experimental validation of the variants identified in the WES study in an independent cohort. We chose NOTCH2 and MYD88 for their known recurrence in SMZL and the genes SMYD1, PDE10A, and ZNF608, which had not been previously described to be mutated in this tumor entity. Pyrosequencing confirmed the somatic origin of these five mutations in the respective discovery case. Moreover, we screened a validation cohort consisting of 24 SMZL FFPE samples for mutations. Pyrosequencing of the same base position previously found mutated in the discovery cohort was used for technical reasons, as quality of FFPE tissue was insufficient for sequencing of the complete coding regions of the genes. Pyrosequencing showed no recurrent variants at NOTCH2 C7310T, SMYD1 G839T, ZNF608 A3659G, PDE10A G1072A while MYD88 T794C was found in 3 out of 24 validation cases (12.5\%, Additional file 7: Figure S1).

Sanger sequencing was used to screen all exons of SMYD1 and the complete PEST domain of NOTCH2 for mutations in those eight patients of the validation cohort where fresh tissue was available. While Sanger sequencing was able to confirm the SNV detected in NOTCH2 in the discovery case itself, no additional mutation within the PEST domain of NOTCH2 was found within the validation cohort (Additional file 8: Figure S2). Including the discovery case, three mutations were detected in SMYD1 (30\% of screened cases) localized in exons 6, 7 and the 3' untranslated region (UTR) (Fig. 3). Sanger sequencing of 


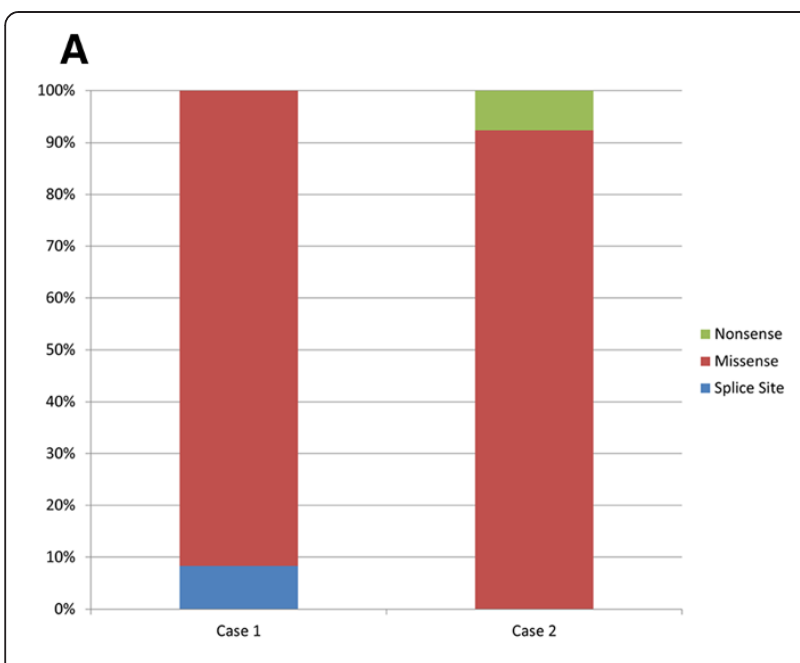

\section{B}

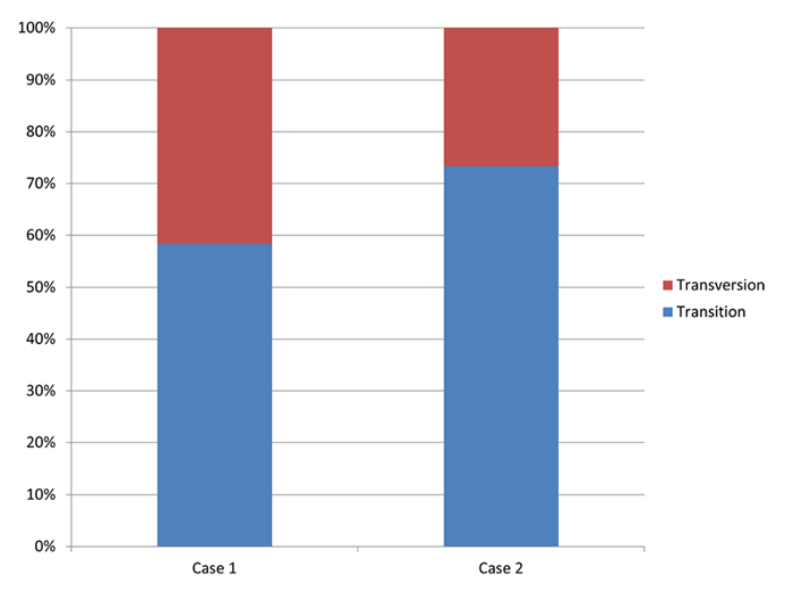

Fig. 1 Distribution of single nucleotide variants in the coding SMZL genome. a. Relative pattern of somatic mutations identified in two SMZL discovery cases using WES. b. Relative distribution of transitions and transversions in discovery cases 1 and 2

non-tumorous germline DNA of each patient was used to ensure the somatic origin of the SMYD1 mutations. We compared our findings to three NGS databases: 1000 genome project [28], Exome Aggregation Consortium database (ExAC, Cambridge, MA [http://exac.broadinstitute.org]), Catalogue of somatic mutations in cancer (COSMIC) [29]. These databases incorporate over 60,000 sequenced exomes with partly overlapping content. The SMYD1 mutations found in our current SMZL cohort are not described in one of the mentioned platforms. SMYD1 seems rather well conserved with only 189 missense or loss-of-funcion variants throughout the gene. COSMIC database contains 170 unique cancer samples with SMYD1 mutations out of 24,615 total samples. Mutations have been discovered in various cancer types (e.g., gastric-, hepatocellular-, bladder-, renal cell carcinoma, melanoma or glioma).

Both exonic mutations are predicted to be damaging by SIFT and Polyphen-2 algorithm and are located within the catalytic SET domain of SMYD1. Moreover, an analysis using Universal Protein Resource (UniProt) by the European Bioinformatics Institute [30] shows that one of the found mutations (c.836G $\geq \mathrm{T} ; \mathrm{p} . \mathrm{C} 279 \mathrm{~F}$ ) is located at one of the four zinc binding sites of the catalytic centre of SMYD1. Furthermore, we used the crystal structure of the murine SMYD1 protein (retrieved from the Protein Data Bank; PDB-ID: 3 N71 $[30,31])$ and localized the detected SMYD1 mutations (Additional file 9: Figure S3). The p.S321Y mutation lies in an exposed position within the functional pocket of the protein. Moreover, the region of the 3' UTR where the SNV was found is predicted to be a high fidelity target of miR-28 (seed length $11, p=0.0007$ ) by the miRWalk platform [32].

\section{Discussion}

This work is the first of its nature to study the whole exome of microdissected SMZL cells. It demonstrates the feasibility of this approach and largely confirms the sequencing results of previous exome sequencing approaches. However, novel mutations in SMZL were discovered, expanding the directory of reported variants. It has to be acknowledged that through bioinformatics selection of putative SNVs using coverage and allele-frequency criteria, significant amounts of mutations might have been lost while falsely positive SNPs may still have been detected. Manual review of all called SNVs was used to avoid such errors.

\section{Clonality of mutations in SMZL}

The number of SMZL analyzed by WES in the current study is small, but laser-microdissection as the method of tumor cell isolation ensures a high purity of tumor DNA for the sequencing. WES with the currently available number of sequencing reads per NGS run has clear limitations in sequencing coverage. Therefore, it is not an ideal method to detect subclonal mutations, especially compared to ultra-deep targeted re-sequencing approaches. Nevertheless, high tumor cell purity may enable also WES to screen for potential subclonal aberrations. We identified mutations that seem to be heterozygous and clonal as they show allele frequencies of $>40 \%$ in the WES and 46-53 \% in the validation experiments (NOTCH2, SMYD1, MYD88), with the proximity to $50 \%$ providing an indirect evidence for high tumor cell purity. However, 12 out of 25 somatic mutations showed mutation frequencies below or equal to $25 \%$, leaving a majority of SNVs likely subclonal variants. This might be a main reason why these genes have not been found as mutated in prior sequencing studies. Although the finding of many presumably subclonal mutations remains to be validated in future in depth WES studies with isolated lymphoma cells, we provide here intial evidence for a 


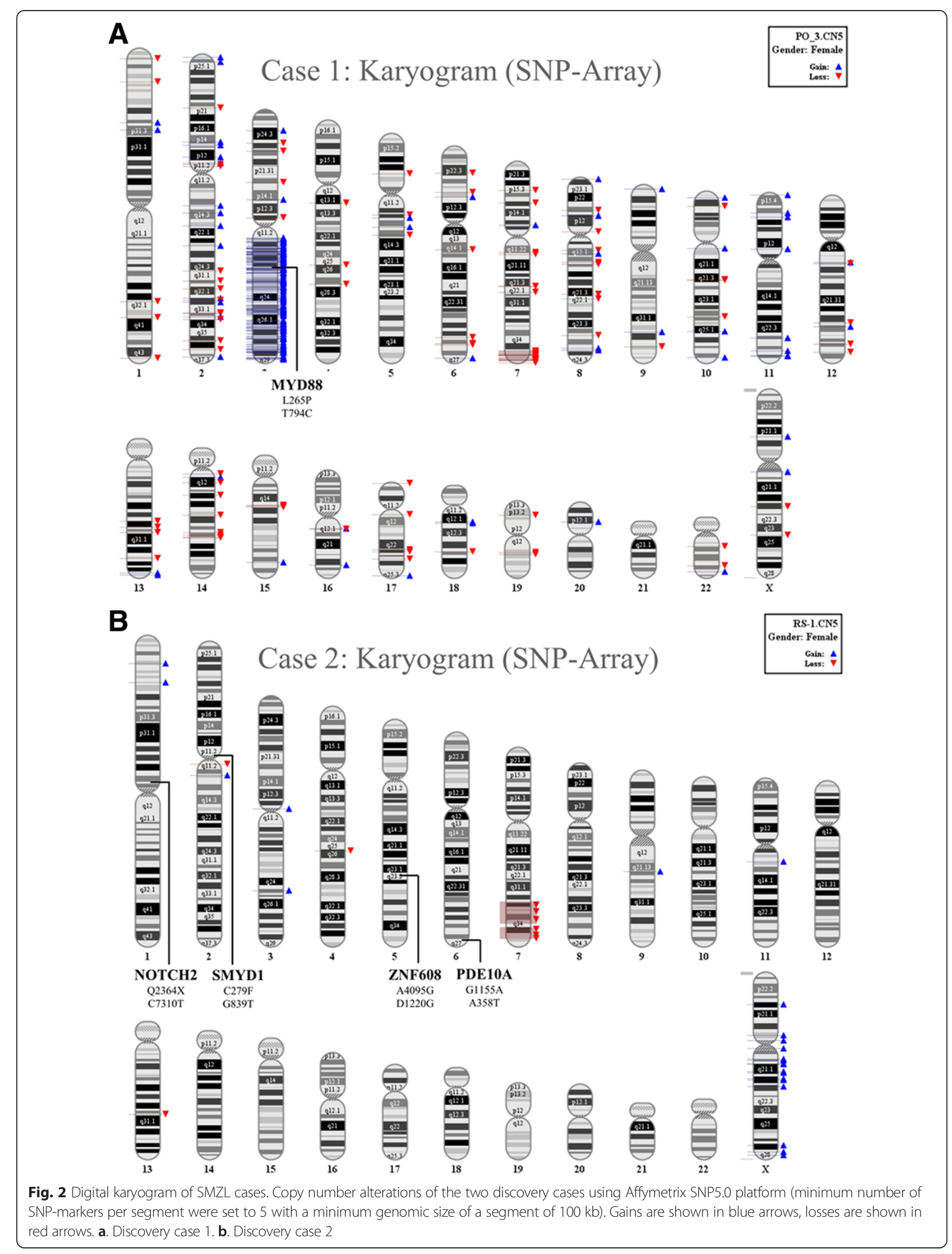




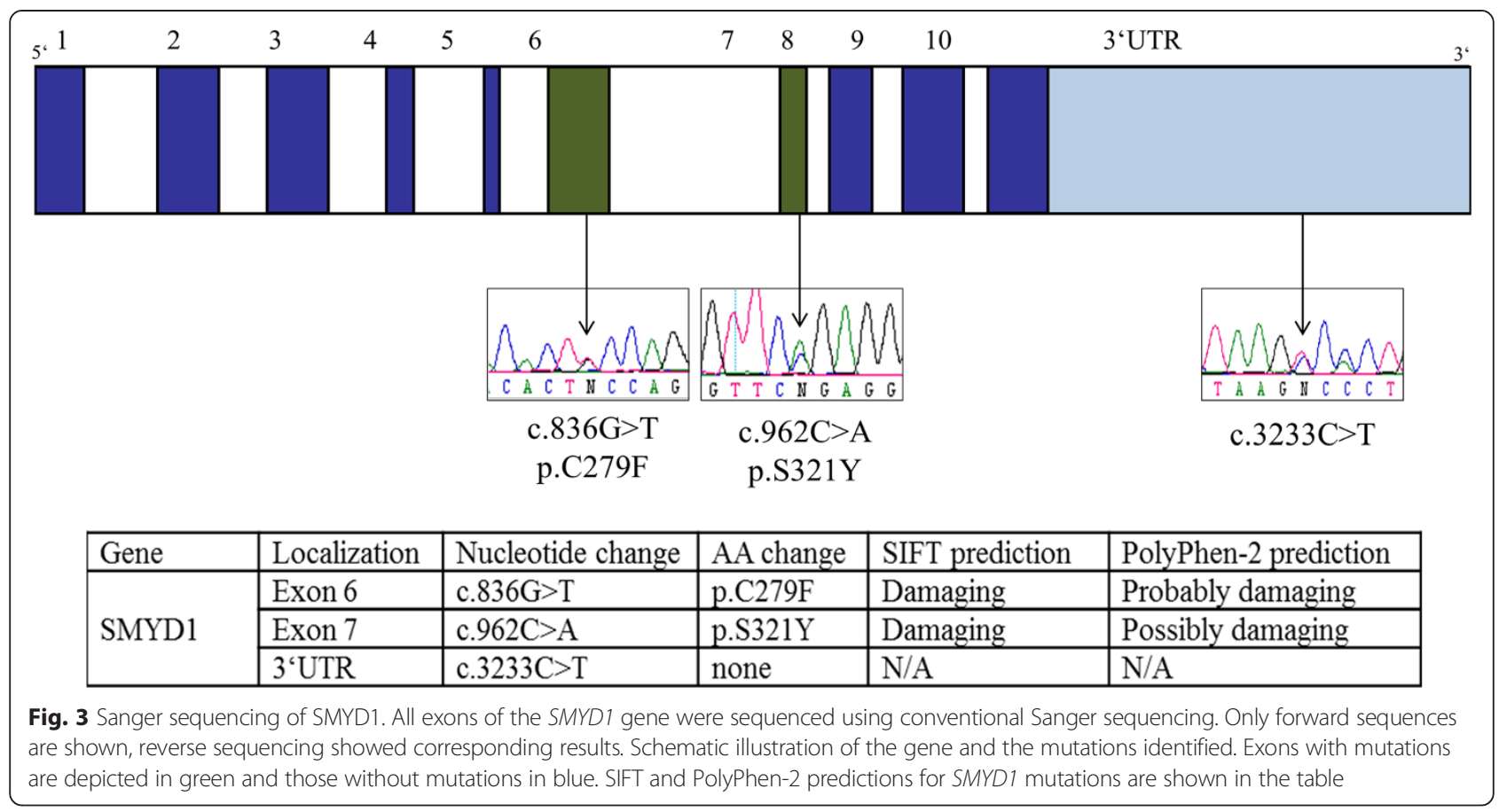

remarkable intraclonal diversification during SMZL clonal expansion.

\section{Mutations in NOTCH2 and MYD88}

Of the 25 non-synonymous variants found within the two discovery cases of the current analysis, NOTCH2 and MYD88 were already known to be recurrently mutated in SMZL [11-14, 23-25]. The heterodimeric transmembrane protein $\mathrm{NOTCH} 2$ has been found mutated in 21-25\% of SMZL by the pivotal studies [11, 14], but in lower frequency in others [12, 13]. NOTCH2 plays an important role in marginal zone B cell development in the spleen and mutations in its pathway were identified in various B-cell lymphomas [33-35]. In our cohort, although present in one of the discovery cases, we did not detect further NOTCH2 mutations at the same base position in the validation cases by pyrosequencing $(1 / 24$ cases, $4.2 \%$ ). As loss-of-function variants, as in NOTCH2, rarely concur at the same base position we sequenced also the functional PEST-domain, still discovering only one variant in ten cases. Discrepancies might be explained by technical issues, e.g., use of FFPE material in studies with lower NOTCH2 mutation frequency. Another explanation could be geographic differences in SMZL etiology, as infectious components like chronic hepatitis $\mathrm{C}$ virus infection are thought to be involved [36].

The cytoplasmatic adaptor MYD88 mediating toll-like receptor induced NF- $\mathrm{B}$ activation has been frequently found mutated in SMZL (10-15\%), in targeted sequencing approaches as well as WES [10, 12, 24, 37].
Especially the L265P missense substitution occurs with high prevalence in various B-cell malignancies [38]. We discovered the L265P variant of MYD88 in 1 of 2 discovery cases and 3 of 24 validation cases in our cohort. The mutation frequency of $15 \%$ is therefore similar to published data.

The transcription factor KLF2, has been found recurrently mutated in SMZL in recent sequencing studies [15-17]. We did not detect any KLF2 mutations. However, large areas of this gene showed poor sequencing coverage, likely due to the high GC content of KLF2. Also the ExAC database shows that throughout 60,706 incorporated exomes there is largely no or minimal coverage of KLF2. Therefore, we might have failed to detect actual KLF2 mutations in our cases.

\section{Novel mutations in SMZL}

It has to be acknowledged that a WES approach as used in the current study can generate a certain statistical amount of false positive findings [39]. We therefore tried to use manual review of mutation sites and multiple online databases as well as literature review to consolidate our findings. Some of the genes bearing mutations in the SMZL cases investigated here, including PRSS1 [40], PCLO [41, 42], CSMD1 [26, 43], HERC2 [44], and MUC12 [45] have been previously described to be mutated in other tumor entities, but not in SMZL. We identified one gene, SMYD1, with recurrent somatic mutations in our collection of SMZL, which has not been specifically associated to cancer in the past. However, other members of the SMYD 
family have putative oncogenic roles. The SMYD family (SMYD1-5) is a group of SET domain-containing transcriptional regulators acting mainly through histone modification [46]. SMYD2 has oncogenic properties by repressing the activity of p53 and RB through methylation $[47,48]$ and SMYD3 is commonly upregulated in hepatocellular carcinoma, colon carcinoma and breast cancer with influence on cell proliferation in vitro [49-52]. SMYD1, which we found mutated in 3 of 10 SMZL cases, has been associated with cell differentiation and embryogenesis of the heart [53]. It acts through the lysine methyltransferase activity of the SET domain, regulating the muscle-specific transcription factor $s k N A C$ and is itself targeted by $M E F 2 C$ during cardiac morphogenesis [54-56]. The two coding mutations found in SMYD1 are both located within this SET domain. The p.C279F mutations is located at one of the four zinc binding sites of the catalytic centre of SMYD1. Furthermore, the p.S321Y mutation projects to the functional pocket of the murine crystal structure of the protein. Interestingly, SMYD1 seems to be involved in NOTCH1 mediated signalling, which could explain a possible involvement in SMZL oncogenesis [57]. Moreover, the position in the 3' UTR of SMYD1, where we found one of the mutations, is a predicted target of miR28, a recently described potent tumor suppressor in B-cell lymphomagenesis [58].

\section{Conclusions}

In this small study, we performed WES and copy number analysis from microdissected SMZL cells. It demonstrates the feasibility of this approach and demonstrates advantages of analysing high tumor cell purity even in times of ultra-deep sequencing. While some mutations found in previous studies could be confirmed, we also expand the reported directory of mutated genes in this lymphoma entity. SMYD1, a gene that has been primarily linked to differentiation and embryogenesis of the heart, was recurrently mutated in our cohort. Similar to closely related genes of the same family (SMYD2, SMYD3), it might play a role in the oncogenesis of SMZL as member of the NOTCH1 signalling pathway. Further functional investigation and studies in larger cohorts will be needed to substantiate this hypothesis.

\section{Additional files}

Additional file 1: Table S1. Clinical characteristics of all SMZL cases. (DOC $29 \mathrm{~kb}$ )

Additional file 2: Table S2. Primers for PCR and pyrosequencing for SNV validation. (DOC $35 \mathrm{~kb}$ )

Additional file 3: Table S3. Primers for Sanger sequencing of the NOTCH2 PEST domain. (DOC $32 \mathrm{~kb}$ )

Additional file 4: Table S4. Primers for Sanger sequencing of SMYD1 all exons. (DOC $50 \mathrm{~kb}$ )
Additional file 5: Table S5. Characteristics of the identified 216 non-synonymous variants. (XLSX $51 \mathrm{~kb}$ )

Additional file 6: Table S6. Coverage statistics for genes of interest. (DOC $30 \mathrm{~kb}$ )

Additional file 7: Figure S1. Pyrosequencing of validation cohort. Five genes with SNVS in the discovery cohort were analysed for recurrent mutations in a validation cohort $(n=24)$. Only the SNV position of the respective discovery case was analysed. A. MYD88 794 T > C. B. NOCTH2 7090C > T. C. SMYD1 836G > T. D. PDE10A 1072 G > A. E. ZNF608 3659A > G. (DOC $291 \mathrm{~kb})$

Additional file 8: Figure S2. Sanger validation of NOTCH2 p.Q2364* nonsense mutation. Nucleotide substitution c.7090C $>$ T in the NOTCH2 PEST domain within exon 34. (DOC $263 \mathrm{~kb}$ )

Additional file 9: Figure S3. Crystalline structure of the murine SMYD1. Coding mutations found in SMZL are highlighted (c.836G > T; P.C279F in blue, c.962C > A; p.S321Y in red). (DOC $232 \mathrm{~kb}$ )

\section{Abbreviations}

FFPE: Formalin-fixed, paraffin-embedded; SMZL: Splenic marginal zone lymphoma; SNP: Single nucleotide polymorphism; SNVs: Single nucleotide variants; UTR: Untranslated region; WES: Whole-exome sequencing.

\section{Competing interests}

The authors have no conflict of interest to declare in context of this manuscript.

\section{Authors' contributions}

J. Peveling-Oberhag designed and performed research, revised and analyzed clinical data and wrote the manuscript; F. Wolters performed research, data analysis and assisted in writing the manuscript; C. Döring performed bioinformatics and statistical analysis; D. Walter revised and analyzed clinical data and revised the paper; L. Sellmann provided clinical samples and performed research; R. Scholtysik performed research, data analysis and revised the paper; M. Lucioni provided histological samples and assisted in the correction of the paper; M. Schubach performed bioinformatics and statistical analysis; M. Paulli provided histological samples and assisted in the correction of the paper; S. Biskup performed research, data analysis and revised the paper; S. Zeuzem designed research, provided advice and revised the paper; R. Küppers designed research, provided advice and revised the paper; ML. Hansmann designed research, provided advice and revised the paper.

\section{Acknowledgement}

We thank Ekaterini Hadzoglou, Sabine Albrecht and Ralf Lieberz for excellent technical assistance. We thank Marc Seifert for assistance with cell sorting. This work was supported by the "Patenschaftsmodell" grant from the medical faculty of the J.W. Goethe-University Hospital, the IFORES program of the Medical Faculty of the University of Duisburg-Essen, the German Ministry of Education and Research (BMBF) through the HaematoSys Project (grant number 0315452G), and by the Deutsche Forschungsgemeinschaft (PE2152/2-1).

\section{Author details}

'Medizinische Klinik 1, Klinikum der Johann Wolfgang Goethe-Universität, Theodor-Stern-Kai 7, Frankfurt am Main, Germany. ${ }^{2}$ Senckenbergisches Institut für Pathologie, Klinikum der Johann Wolfgang Goethe-Universität, Theodor-Stern-Kai 7, Frankfurt am Main, Germany. Institute of Cell Biology (Cancer Research), Medical School, University of Duisburg-Essen, Essen, Germany. ${ }^{4}$ Department of Human Pathology, Fondazione IRCCS Policlinico San Matteo, University of Pavia, Pavia, Italy. ${ }^{5}$ Institute of Medical Genetics and Human Genetics, Charité Universitätsmedizin Berlin, Augustenburger Platz 1, Berlin, Germany. ${ }^{6} \mathrm{CeGaT}$ GmbH, Paul-Ehrlich-Straße 23, Tübingen, Germany. ${ }^{7}$ German Cancer Consortium (DKTK), Heidelberg, Germany.

Received: 6 April 2015 Accepted: 10 October 2015

Published online: 24 October 2015

\section{References}

1. Swerdlow SH, Campo E, Harris NL, Pileri SA, Stein H, Thiele J, et al. WHO classification of tumours of haematopoietic and lymphoid tissues. Lyon: IARC Press; 2008. 
2. Dufresne SD, Felgar RE, Sargent RL, Surti U, Gollin SM, McPhail ED, et al. Defining the borders of splenic marginal zone lymphoma: a multiparameter study. Hum Pathol. 2010;41(4):540-51.

3. Dungarwalla M, Appiah-Cubi S, Kulkarni S, Saso R, Wotherspoon A, Osuji N, et al. High-grade transformation in splenic marginal zone lymphoma with circulating villous lymphocytes: the site of transformation influences response to therapy and prognosis. Br J Haematol. 2008;143(1):71-4.

4. Salido M, Baro C, Oscier D, Stamatopoulos K, Dierlamm J, Matutes E, et al. Cytogenetic aberrations and their prognostic value in a series of 330 splenic marginal zone B-cell lymphomas: a multicenter study of the Splenic B-Cell Lymphoma Group. Blood. 2010;116(9):1479-88.

5. Robledo C, Garcia JL, Benito R, Flores T, Mollejo M, Martinez-Climent JA, et al. Molecular characterization of the region 7q22.1 in splenic marginal zone lymphomas. PLoS One. 2011;6(9):e24939.

6. Watkins AJ, Hamoudi RA, Zeng N, Yan Q, Huang Y, Liu H, et al. An integrated genomic and expression analysis of $7 q$ deletion in splenic marginal zone lymphoma. PLoS One. 2012;7(9), e44997.

7. Arribas AJ, Gomez-Abad C, Sanchez-Beato M, Martinez N, Dilisio L, Casado F, et al. Splenic marginal zone lymphoma: comprehensive analysis of gene expression and miRNA profiling. Mod Pathol: Off J US Canadian Acad Pathol Inc. 2013;26(7):889-901.

8. Novak U, Rinaldi A, Kwee I, Nandula SV, Rancoita PM, Compagno M, et al. The NF-\{kappa\}B negative regulator TNFAIP3 (A20) is inactivated by somatic mutations and genomic deletions in marginal zone lymphomas. Blood. 2009;113(20):4918-21.

9. Peveling-Oberhag J, Crisman G, Schmidt A, Doring C, Lucioni M, Arcaini L, et al. Dysregulation of global microRNA expression in splenic marginal zone lymphoma and influence of chronic hepatitis C virus infection. Leukemia. 2012;26(7):1654-62.

10. Rossi D, Deaglio S, Dominguez-Sola D, Rasi S, Vaisitti T, Agostinelli C, et al. Alteration of BIRC3 and multiple other NF-kappaB pathway genes in splenic marginal zone lymphoma. Blood. 2011;118(18):4930-4.

11. Kiel MJ, Velusamy T, Betz BL, Zhao L, Weigelin HG, Chiang MY, et al. Wholegenome sequencing identifies recurrent somatic NOTCH2 mutations in splenic marginal zone lymphoma. J Exp Med. 2012;209(9):1553-65.

12. Martinez N, Almaraz C, Vaque JP, Varela I, Derdak S, Beltran S, et al. Wholeexome sequencing in splenic marginal zone lymphoma reveals mutations in genes involved in marginal zone differentiation. Leukemia. 2014;28(6):1334-40.

13. Parry M, Rose-Zerilli MJ, Gibson J, Ennis S, Walewska R, Forster J, et al. Whole exome sequencing identifies novel recurrently mutated genes in patients with splenic marginal zone lymphoma. PLoS One. 2013;8(12), e83244.

14. Rossi D, Trifonov V, Fangazio M, Bruscaggin A, Rasi S, Spina V, et al. The coding genome of splenic marginal zone lymphoma: activation of NOTCH2 and other pathways regulating marginal zone development. J Exp Med. 2012;209(9):1537-51.

15. Clipson A, Wang M, de Leval L, Ashton-Key M, Wotherspoon A, Vassiliou G, et al. KLF2 mutation is the most frequent somatic change in splenic marginal zone lymphoma and identifies a subset with distinct genotype. Leukemia. 2015;29(5):1177-85.

16. Piva R, Deaglio S, Fama R, Buonincontri R, Scarfo I, Bruscaggin A, et al. The Kruppel-like factor 2 transcription factor gene is recurrently mutated in splenic marginal zone lymphoma. Leukemia. 2015;29(2):503-7.

17. Parry M, Rose-Zerilli MJ, Ljungstrom V, Gibson J, Wang J, Walewska R, et al. Genetics and Prognostication in Splenic Marginal Zone Lymphoma: Revelations from Deep Sequencing. Clin Cancer Res. 2015.

18. Swerdlow SH, International Agency for Research on Cancer, World Health Organization. WHO classification of tumours of haematopoietic and lymphoid tissues. 4th ed. Lyon: International Agency for Research on Cancer; 2008.

19. Grant JR, Arantes AS, Liao X, Stothard P. In-depth annotation of SNPS arising from resequencing projects using NGS-SNP. Bioinformatics. 2011;27(16):2300-1.

20. Flicek P, Amode MR, Barrell D, Beal K, Brent S, Carvalho-Silva D, et al. Ensembl 2012. Nucleic Acids Res. 2012;40(Database issue):D84-90.

21. Hubbard T, Barker D, Birney E, Cameron G, Chen Y, Clark L, et al. The Ensembl genome database project. Nucleic Acids Res. 2002;30(1):38-41.

22. Robinson JT, Thorvaldsdottir H, Winckler W, Guttman M, Lander ES, Getz G, et al. Integrative genomics viewer. Nat Biotechnol. 2011;29(1):24-6.

23. Yan Q, Huang Y, Watkins AJ, Kocialkowski S, Zeng N, Hamoudi RA, et al. BCR and TLR signaling pathways are recurrently targeted by genetic changes in splenic marginal zone lymphomas. Haematologica. 2012;97(4):595-8.

24. Troen G, Warsame A, Delabie J. CD79B and MYD88 Mutations in Splenic Marginal Zone Lymphoma. ISRN Oncology. 2013;2013:252318.
25. Troen G, Nygaard V, Jenssen TK, Ikonomou IM, Tierens A, Matutes E, et al Constitutive expression of the AP-1 transcription factors c-jun, junD, junB, and c-fos and the marginal zone B-cell transcription factor Notch2 in splenic marginal zone lymphoma. JMD. 2004;6(4):297-307.

26. Kumar P, Henikoff S, Ng PC. Predicting the effects of coding non-synonymous variants on protein function using the SIFT algorithm. Nat Protoc. 2009;4(7):1073-81.

27. Adzhubei IA, Schmidt S, Peshkin L, Ramensky VE, Gerasimova A, Bork P, et al. A method and server for predicting damaging missense mutations. Nat Methods. 2010;7(4):248-9.

28. Genomes Project C, Abecasis GR, Altshuler D, Auton A, Brooks LD, Durbin RM, et al. A map of human genome variation from population-scale sequencing. Nature. 2010;467(7319):1061-73.

29. Forbes SA, Beare D, Gunasekaran P, Leung K, Bindal N, Boutselakis $H$, et al. COSMIC: exploring the world's knowledge of somatic mutations in human cancer. Nucleic Acids Res. 2015;43(Database issue):D805-11.

30. UniProt C. UniProt: a hub for protein information. Nucleic Acids Res. 2015;43(Database issue):D204-12.

31. Sirinupong N, Brunzelle J, Ye J, Pirzada A, Nico L, Yang Z. Crystal structure of cardiac-specific histone methyltransferase SmyD1 reveals unusual active site architecture. J Biol Chem. 2010;285(52):40635-44.

32. Dweep H, Sticht C, Pandey P, Gretz N. miRWalk-database: prediction of possible miRNA binding sites by "walking" the genes of three genomes. J Biomed Inform. 2011:44(5):839-47.

33. Kridel R, Meissner B, Rogic S, Boyle M, Telenius A, Woolcock B, et al. Whole transcriptome sequencing reveals recurrent NOTCH1 mutations in mantle cell lymphoma. Blood. 2012;119(9):1963-71.

34. Puente XS, Pinyol M, Quesada V, Conde L, Ordonez GR, Villamor N, et al. Whole-genome sequencing identifies recurrent mutations in chronic lymphocytic leukaemia. Nature. 2011;475(7354):101-5.

35. Fabbri G, Rasi S, Rossi D, Trifonov V, Khiabanian H, Ma J, et al. Analysis of the chronic lymphocytic leukemia coding genome: role of NOTCH1 mutational activation. J Exp Med. 2011;208(7):1389-401.

36. Peveling-Oberhag J, Arcaini L, Hansmann ML, Zeuzem S. Hepatitis C-associated B-cell non-Hodgkin lymphomas. Epidemiology, molecular signature and clinical management. J Hepatol. 2013;59(1):169-77.

37. Varettoni M, Arcaini L, Zibellini S, Boveri E, Rattotti S, Riboni R, et al. Prevalence and clinical significance of the MYD88 (L265P) somatic mutation in Waldenstrom's macroglobulinemia and related lymphoid neoplasms. Blood. 2013;121(13):2522-8.

38. Ngo VN, Young RM, Schmitz R, Jhavar S, Xiao W, Lim KH, et al. Oncogenically active MYD88 mutations in human lymphoma. Nature. 2011;470(7332):115-9.

39. Lawrence MS, Stojanov P, Polak P, Kryukov GV, Cibulskis K, Sivachenko A, et al. Mutational heterogeneity in cancer and the search for new cancerassociated genes. Nature. 2013;499(7457):214-8.

40. Zeng K, Liu QC, Lin JH, Lin XH, Zhuang ZH, Gao F, et al. Novel mutations of PRSS1 gene in patients with pancreatic cancer among Han population. Chin Med J. 2011;124(13):2065-7.

41. Zhang Y, Qiu Z, Wei L, Tang R, Lian B, Zhao Y, et al. Integrated analysis of mutation data from various sources identifies key genes and signaling pathways in hepatocellular carcinoma. PLoS One. 2014;9(7), e100854.

42. Lohr JG, Stojanov P, Lawrence MS, Auclair D, Chapuy B, Sougnez C, et al. Discovery and prioritization of somatic mutations in diffuse large B-cell lymphoma (DLBCL) by whole-exome sequencing. Proc Natl Acad Sci U S A. 2012;109(10):3879-84.

43. Farrell C, Crimm H, Meeh P, Croshaw R, Barbar T, Vandersteenhoven JJ, et al. Somatic mutations to CSMD1 in colorectal adenocarcinomas. Cancer Biol Ther. 2008;7(4):609-13.

44. Yoo NJ, Park SW, Lee SH. Frameshift mutations of ubiquitination-related genes HERC2, HERC3, TRIP12, UBE2Q1 and UBE4B in gastric and colorectal carcinomas with microsatellite instability. Pathology. 2011;43(7):753-5.

45. Nichols AC, Chan-Seng-Yue M, Yoo J, Xu W, Dhaliwal S, Basmaji J, et al. A Pilot Study Comparing HPV-Positive and HPV-Negative Head and Neck Squamous Cell Carcinomas by Whole Exome Sequencing. ISRN Oncology. 2012;2012:809370.

46. Leinhart K, Brown M. SET/MYND Lysine Methyltransferases Regulate Gene Transcription and Protein Activity. Genes. 2011;2(1):210-8.

47. Saddic LA, West LE, Aslanian A, Yates 3rd JR, Rubin SM, Gozani O, et al. Methylation of the retinoblastoma tumor suppressor by SMYD2. J Biol Chem. 2010;285(48):37733-40. 
48. Huang J, Perez-Burgos L, Placek BJ, Sengupta R, Richter M, Dorsey JA, et al. Repression of p53 activity by Smyd2-mediated methylation. Nature. 2006;444(7119):629-32.

49. Hamamoto R, Furukawa Y, Morita M, limura Y, Silva FP, Li M, et al. SMYD3 encodes a histone methyltransferase involved in the proliferation of cancer cells. Nat Cell Biol. 2004;6(8):731-40.

50. Hamamoto R, Silva FP, Tsuge M, Nishidate T, Katagiri T, Nakamura Y, et al. Enhanced SMYD3 expression is essential for the growth of breast cancer cells. Cancer Sci. 2006;97(2):113-8.

51. Lin YM, Furukawa Y, Tsunoda T, Yue CT, Yang KC, Nakamura Y. Molecular diagnosis of colorectal tumors by expression profiles of 50 genes expressed differentially in adenomas and carcinomas. Oncogene. 2002;21(26):4120-8.

52. Okabe H, Satoh S, Kato T, Kitahara O, Yanagawa R, Yamaoka Y, et al. Genome-wide analysis of gene expression in human hepatocellular carcinomas using CDNA microarray: identification of genes involved in viral carcinogenesis and tumor progression. Cancer Res. 2001;61(5):2129-37.

53. Gottlieb PD, Pierce SA, Sims RJ, Yamagishi H, Weihe EK, Harriss JV, et al. Bop encodes a muscle-restricted protein containing MYND and SET domains and is essential for cardiac differentiation and morphogenesis. Nat Genet. 2002;31(1):25-32

54. Phan D, Rasmussen TL, Nakagawa O, McAnally J, Gottlieb PD, Tucker PW, et al. BOP, a regulator of right ventricular heart development, is a direct transcriptional target of MEF2C in the developing heart. Development 2005;132(11):2669-78.

55. Sims 3rd RJ, Weihe EK, Zhu L, O'Malley S, Harriss JV, Gottlieb PD. m-Bop, a repressor protein essential for cardiogenesis, interacts with skNAC, a heartand muscle-specific transcription factor. J Biol Chem. 2002;277(29):26524-9.

56. Tan X, Rotllant J, Li H, De Deyne P, Du SJ. SmyD1, a histone methyltransferase, is required for myofibril organization and muscle contraction in zebrafish embryos. Proc Natl Acad Sci U S A. 2006;103(8):2713-8.

57. Kwon C, Qian L, Cheng P, Nigam V, Arnold J, Srivastava D. A regulatory pathway involving Notch1/beta-catenin/Isl1 determines cardiac progenitor cell fate. Nat Cell Biol. 2009;11(8):951-7.

58. Schneider C, Setty M, Holmes AB, Maute RL, Leslie CS, Mussolin L, et al. MicroRNA 28 controls cell proliferation and is down-regulated in B-cell lymphomas. Proc Natl Acad Sci U S A. 2014;111(22):8185-90.

\section{Submit your next manuscript to BioMed Central and take full advantage of:}

- Convenient online submission

- Thorough peer review

- No space constraints or color figure charges

- Immediate publication on acceptance

- Inclusion in PubMed, CAS, Scopus and Google Scholar

- Research which is freely available for redistribution 